\title{
Tuberculum Sellae Meningiomas: Evolving Surgical Strategies
}

\author{
Shaan M. Raza $\cdot$ Sabih T. Effendi • \\ Franco DeMonte
}

Published online: 24 September 2014

(C) Springer Science + Business Media New York 2014

\begin{abstract}
Tuberculum sella meningiomas represent a unique set of surgical challenges due to their three-dimensional relationship with the optic apparatus, critical vasculature, and the pituitary stalk. These challenges are encountered with the context of a surgical goal that provides long-term tumor control, preservation or improvement of visual function and minimal morbidity. Advancements in skull base surgery with improvements in transcranial (TCR) approaches and endoscopic endonasal approaches have added to the surgical strategies available. We review the modern literature and compare techniques and their outcomes in an effort to determine the relative merits and indications of TCR and endoscopic endonasal approaches in the management of this set of tumors.
\end{abstract}

Keywords Tuberculum sellae $\cdot$ Meningioma $\cdot$ Skull base . Open skull base $\cdot$ Endoscopic endonasal skull base . Minimally invasive $\cdot$ Surgery

$\begin{array}{ll}\text { Abbreviations } \\ \text { TSM } & \text { Tuberculum sella meningiomas } \\ \text { TCR } & \text { Transcranial } \\ \text { EEA } & \text { Expanded endonasal approaches }\end{array}$

This article is part of the Topical Collection on Brain Tumor Surgery.

S. M. Raza $(\bowtie) \cdot$ S. T. Effendi · F. DeMonte Department of Neurosurgery, The University of Texas MD Anderson Cancer Center, P.O. Box 301402-442, Houston, TX 77230-1402, USA

e-mail: SMRaza@mdanderson.org

\section{Introduction}

Meningiomas are the most common intracranial tumor, accounting for $32 \%$ of all brain tumors [1]. Tuberculum sella meningiomas (TSM) represent $4-10 \%$ of this entire cohort and are considered one of the more common intracranial meningiomas [1,2]. TSMs most commonly present with insidious and progressive visual symptoms, including visual loss (98\%), optic nerve atrophy (78 \%), and visual field deficits [3]. Visual loss can present in different patterns. A unilateral temporal field defect with a contralateral quadrantanopsia is the most common presentationoccurring in nearly two-thirds in some published series [1$3,4 \bullet, 5]$. Patients can also present with a chiasmal syndrome, with optic nerve atrophy, asymmetric bitemporal visual field deficits, and a non-expanded sella [8]. FosterKennedy syndrome, defined as ipsilateral optic atrophy and contralateral papilledema, occurs in $5 \%$ of patients [3]. Other symptoms include hemiparesis $(15 \%)$ and anosmia (11\%) [3].

Histologically, meningiomas appear as sheets of densely pack cells with whorls and psammoma bodies; however, numerous histologic subtypes have been described [5]. The 2007 World Health Organization (WHO) classifies meningiomas into three grades: I (low grade), II (atypical), and III (anaplastic) [5]. Overall recurrence rates increase with higher WHO grade: $9 \%$ for grade I, $29 \%$ for atypical, and $50 \%$ for anaplastic [6]. There is no data to suggest that the histological behavior of TSM is different from such tumors at other anatomic sites.

While their biological behavior may not differ, TSMs are characterized by a unique set of anatomic considerations that dictate relevant treatment outcomes and decision-making paradigms. Recent developments in skull base surgery-improvements in microsurgical technique, the 
introduction of expanded endonasal techniques, stereotactic radiosurgery-warrant of an overview of the different techniques and the reported outcomes in the literature.

\section{Natural History and Decision to Treat}

\section{Management}

Several natural history studies of incidentally discovered meningiomas indicate several patterns of growth. Oya et al. demonstrated in a cohort of 244 patients with meningiomas (skull base and non-skull base) followed over a mean of 3.8 years that $44 \%$ of patients exhibited linear growth while the remaining demonstrated a complex growth pattern [7]. Hashiba et al. similarly demonstrated the need for long-term surveillance in incidentally discovered meningiomas [8]. Factors at presentation linked with future growth included: age 60 or younger, absence of calcification, MRI T2 signal hyperintensity, and peritumoral edema In comparing incidentally discovered with symptomatic meningiomas, Hashimoto et al. demonstrated that nonsymptomatic lesions tended to have a significantly lower mean MIB-1 index-further bolstering the need to intervene with symptomatic lesions at presentation [9•].

Given the existing natural history data of meningiomas, all patients presenting with TSM undergo formal neuroophthalmological evaluation in addition to standard imaging. Evidence of subjective or objective correlating symptoms and/or compression of the optic apparatus are indications for surgical intervention. In those patients presenting with small incidentally discovered lesions, an option is to monitor closely with imaging and neuro-ophthalmology evaluations in an effort to capture any nonlinear growth. Evidence of subsequent growth and/or the development of symptoms are an indication for subsequent intervention.

\section{Surgical Anatomy and Operative Considerations}

The sellae turcica (pituitary fossa) lies in the body of the sphenoid bone, bounded anteriorly by the tuberculum sellae and posteriorly by the dorsum sellae [10]. Anterior to the tuberculum sellae lies the planum sphenoidale. Between these two structures is a shallow groove, the chiasmatic sulcus (groove). The optic foramen with the optic nerves are lateral to the chiasmatic sulcus. The optic chiasm lies posterior and superior to the sulcus. The limbus sphenoidalis is a ridge of bone at the anterior aspect of the chiasmatic sulcus. TSMs arise primarily from the dura covering the tuberculum sellae and chiasmatic sulcus, and can involve the dura of the limbus sphenoidalis anteriorly and/or diaphragma sellae posteriorly [11•]. Given their anatomic site of origin, the blood supply to TSM typically is from the posterior ethmoidal arteries; with increasing size, arterial supply is parasitized from the anterior communicating artery (AComm) and anterior cerebral arteries.

As these tumors grow, they displace and can encompass nearby structures. The tendency for these tumors to distort adjacent anatomy can be quite dramatic. The optic nerves may even be displaced lateral to the carotid arteries. Also important to note is the potential for extension into the sella. With classic TSM, the diaphragma sella provides a barrier and is commonly displaced inferiorly. Those tumors arising from the diaphragm sella can often have gross tumor extension into the sella turcica. The pituitary stalk can be adherent along the posterior aspect of the capsule but is generally easy to locate due to its vascular and fleshy appearance. Due to its characteristic appearance, the risk of iatrogenic pituitary dysfunction is low. Identification and preservation of the superior hypophyseal arteries, which typically lie along the lateral aspects of the tumor, is imperative for this reason.

While imaging findings may indicate posterior encasement of the basilar apex and its branches, the membrane of Liliquist provides an arachnoidal barrier from this critical vasculature. The absence or scarring of such arachnoidal membranes from prior radiation or surgery can increase the risk of injury to adjacent structures. Larger tumors may displace or encase the supraclinoid internal carotid artery (ICA) laterally and the anterior cerebral artery (ACA)AComm complex superiorly. During resection, these arteries (including the recurrent artery of Huebner) must be identified and tumor must carefully be dissected away from these arteries and their branches. The A2 segments of the anterior cerebral arteries are commonly separated from the superior-posterior pole of the tumor by the arachnoid of the interhemispheric fissure.

With growth, the tumor displaces the optic nerves laterally and the chiasm posterior-superiorly. The relationship of the chiasm in relation to TSM is characteristic and differentiates them from planum sphenoidale and olfactory groove meningiomas. An important feature of TSMs is the tendency to extend into the optic canals. A retrospective review by Pamir et al. [12] correlated the presence of marked asymmetric visual findings with the presence of optic canal invasion; hence, such clinical findings on preoperative ophthalmological exams warrants intraoperative canal unroofing and exploration. In addition, studies have demonstrated that meningioma invasion into the optic canals can be higher than anticipated preoperatively on the basis of MRI [13]. Preoperative impairment of vision can be the result of long-term compression, thinning, and demyelination-as a result, the optic nerves within and outside of the canal are at risk for surgical injury [14]. Given one of the relevant surgical outcomes is visual 

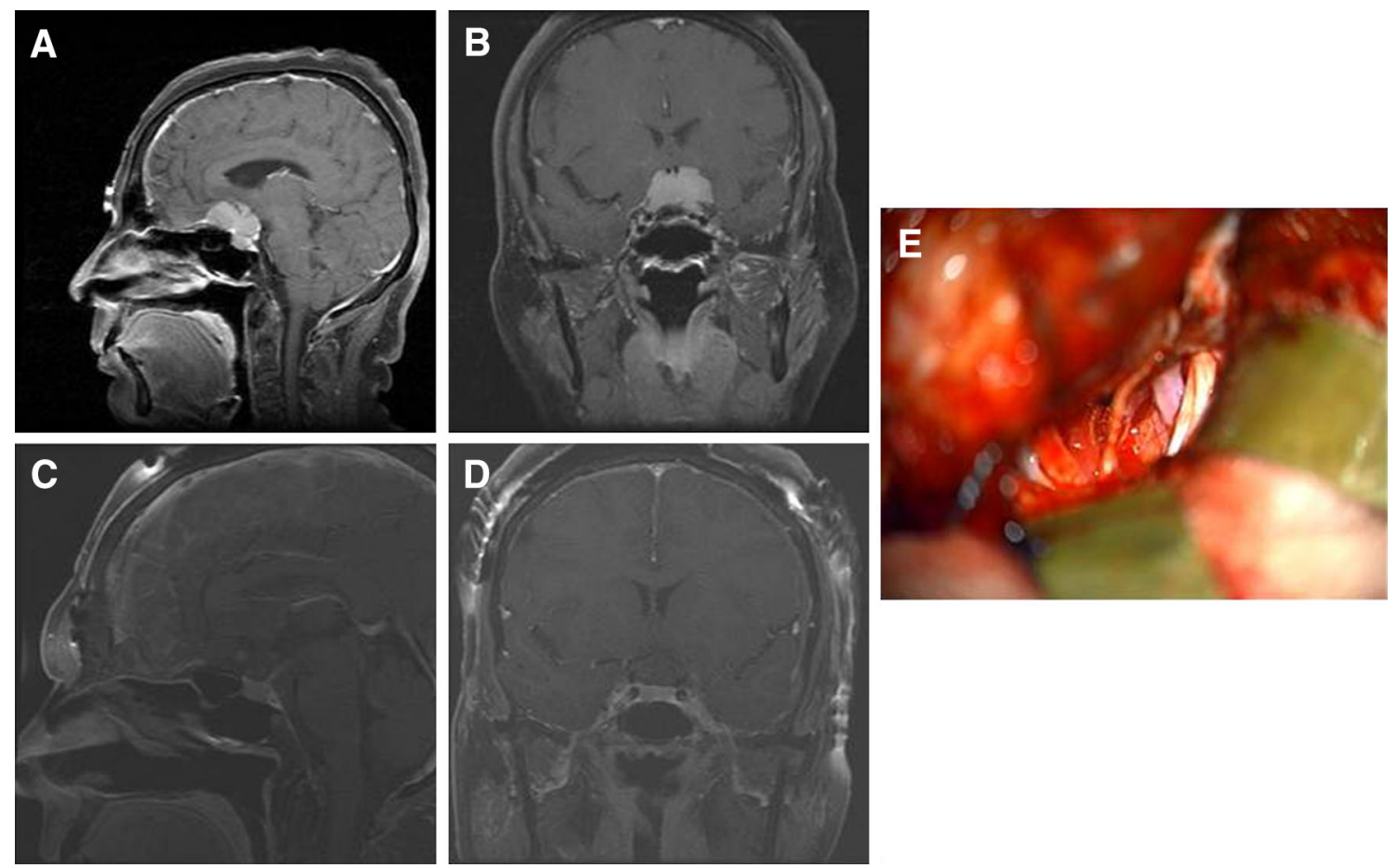

Fig. 1 Case example of tuberculum sella meningioma resected via orbitocranial craniotomy. Panels $\mathbf{a}$ and $\mathbf{b}$ are preoperative contrastenhanced MRI T1-weighted scans demonstrating a tuberculum sella meningioma with bilateral optic canal extension and involvement of

function, protection of the optic apparatus and its appropriate decompression is essential in any operation. While preoperative ophthalmological examination may demonstrate optic disc pallor, even partially demyelinated optic nerves may remyelinate after decompression of the optic apparatus. Consequently, one of the crucial steps in any resection-regardless of approach-is unroofing of the optic canal. Beyond canal invasion, tumor-induced hyperostosis is another mechanism of preoperative visual decline that is addressed with canal unroofing. In a retrospective cohort analysis, Nozaki et al. [15] have demonstrated that canal unroofing was an independent factor prognostic of postoperative visual function. In a series of 23 patients operated on via transcranial (TCR) approaches, Sade and Lee demonstrated a $78 \%$ improvement in visual outcomes with canal unroofing and intracanalicular tumor resection [16]. Several reports have also indicated that long-term recurrences of TSMs can be attributed to residual tumor inadvertently left within the optic canals [17].

\section{Transcranial Approach for Resection}

\section{Technique Overview}

A spectrum of surgical approaches have been described in the management of TSMs and can generally be classified as the anterior communicating artery complex. Panels $\mathbf{c}$ and $\mathbf{d}$ are postoperative scans demonstrating a gross total resection. Panel $\mathbf{e}$ is an intraoperative image showing both optic nerves, the pituitary stalk and the left supraclinoid internal carotid artery

anteriorly based (i.e., orbitocranial, supraorbital, and bifrontal) or anterolaterally based (i.e., pterional and modified orbitozygomatic) craniotomies [18-20]. More recently, "minimally invasive" keyhole craniotomies, such as the transciliary or transpalpeberal supraorbital craniotomy, have been described. While they provide theoretical cosmetic advantages, they are limited in their ability to deal with bilateral optic canal involvement or extensive vascular involvement [18-20] (Fig. 1).

While these techniques have been reported extensively in the literature, we will discuss the relevant aspects of our technique. A unilateral orbitocranial craniotomy is typically performed with removal of the orbital rim in order to increase surgeon working space. This is performed via a bicoronal or hemicoronal incision in order to harvest a wide pericranial flap in case the sphenoid sinus is entering during drilling of involved bone in the planum or tuberculum. After performing the craniotomy, an extradural unroofing of the optic canal can be performed. This is done by resecting the posterior orbital roof, identifying the intraorbital end of the optic canal, performing an anterior clinoidectomy and then finally performing a $270^{\circ}$ decompression of the canal. An alternative to an extradural optic canal decompression is to perform this intradurally prior to any manipulation of the optic nerve or tumor. After epidural hemostasis and dural opening, the frontal lobe is dissected free from the ipsilateral olfactory nerve and the proximal sylvian fissure is opened for CSF 
relaxation and mobilization of the frontal lobe away from the anterior skull base. The insertion of the falx into the crista galli marks midline and is followed back to the tumor. An initial midline trajectory is employed to devascularize and debulk tumor starting at the planum sphenoidale and working posteriorly along the tuberculum toward the diaphragm sella. As the tumor is debulked and dissected along an arachnoidal plane, the ipsilateral optic nerve, which may have been initially stretched to the point of translucency, can be identified. If not done extradurally, the optic canal is unroofed with the use of a diamond drill after incising the falciform ligament and creating a dural flap. Under copious irrigation, the canal bone is thinned down to an egg-shell and the remaining bone removed with microcurrettes. As tumor resection proceeds, the ipsilateral optic nerve is followed in order to identify the chiasm, contralateral optic nerve, and the anterior cerebral arteries (and its pertinent branches) which will lie superior to the neural structures. Additional vascular structures that must be identified early include the supraclinoid carotid, ophthalmic artery, posterior communicating artery, and anterior choroidal artery. As the optic apparatus is dissected, subchiasmatic branches from the superior hypophyseal arteries and ICA branches to the optic nerves must been preserved. While working in the subchiasmatic space, the pituitary stalk is identified toward the end of the resection when dissecting the posterior aspect of the tumor; the membrane of Lilliquist serves as a useful dissection plane to identify and follow subsequently in order to protect the basilar apex. When dissecting around the optic nerve within the canal, the vasculature of the nerve should be preserved, including the ophthalmic and central retinal arteries. In cases of bony invasion, any involved bone is drilled out with the aid of stereotactic-guide CT imaging. If the sphenoid sinus is entered, fat from the abdomen can be used to pack the sinus and a large pericranial flap is placed over the defect. Dural closure, bone flap placement and soft tissue closure subsequently are done in a standard fashion.

\section{Selected Case Series: Extent of Resection,}

Complications, Outcomes, and Recurrence

Select large case series on resection of TSMs via a TCA in the modern era are summarized in Table $1[1,2,4 \bullet, 21]$. These surgical series consisted of symptomatic patients and/or those with large lesions, without selection bias toward the size, lateral extensions, optic canal involvement, or encasement of arteries. Gross total resection rates (Simpson grade I or II) ranged from 84 to $100 \%$ and recurrence rates ranged from 0 to $8 \%$. Complications included: CSF leak (range 1-7\%), anosmia (5-6\%), hemorrhage (2-10\%), infection (2-4\%), stroke (1-2\%), vascular injury (0-6\%), and death (1-3\%). Transient DI occurred in 0-3\% of patients and $0 \%$ of patients experienced anterior pituitary dysfunction. Postoperatively, vision improved in 64-74\% of patients, remained stable in 16-26\% of patients, and worsened in $8-12 \%$ of patients.

According to these series, there is a significant increase in complication rates-including suboptimal visual outcomes-with tumor sizes $>3 \mathrm{~cm}$. At this size threshold, there is likely more extensive involvement of ACA branches (and perforators), pial invasion, and compression of the optic apparatus (with its blood supply) that increases the risk of resection. In addition, predictors of poor postoperative visual outcome include either optic disc pallor or optical computed tomography (OCT) measures indicative of nerve degeneration on preoperative testing. From the technical standpoint, optic canal unroofing early during resection is an independent factor that is positively prognostic of improved postoperative visual function. Overall, in comparison to older studies with reported mortality rates of up to $44 \%$ [22], more recent series demonstrate that complex tumors with vascular involvement and extension along the canalicular portion of the optic nerves can be resected with good long-term tumor control, visual outcomes, and reduced morbidity.

\section{Endoscopic Endonasal Approach for Resection}

\section{Technique Overview}

The general aspects of the endoscopic endonasal approach have been reported in the literature [11•]; the nuances will be discussed. Given that the intraoperative CSF leak rate is $100 \%$, we place a lumbar drain after anesthesia induction and prior to head positioning; this remains clamped during surgery and is used for CSF diversion for $24 \mathrm{~h}$ postoperatively. During the nasal stage of the operation, we perform bilateral posterior ethmoidectomies to ensure access to the anterior most aspect of the tumor along the planum sphenoidale with a $0^{\circ}$ endoscope. The middle turbinates (preferably the left one) are resected in selected cases based on the width of the corridor. A Hadad-Bassagasteguy vascularized nasal septal flap is harvested in all cases. After completion of a wide sphenoidotomy that extends laterally to the pterygoid plates, the sphenoid sinus septa are taken down flush in order to identify key bony landmarks (medial opticocarotid recess, clinoidal carotid artery, medial cavernous sinus, optic canals, and tuberculum). With the aid of neuronavigation, a high speed drill under constant irrigation is used to remove bone over the sella, medial OCR, medial cavernous sinus, tuberculum sella, and planum. The extent of optic canal unroofing is dependent on the degree to which there is tumor extension into the canal-this can be determined by preoperative MR imaging. With the use of laser Doppler, the position of the clinoidal carotid arteries and ophthalmic arteries are verified. The dura is 


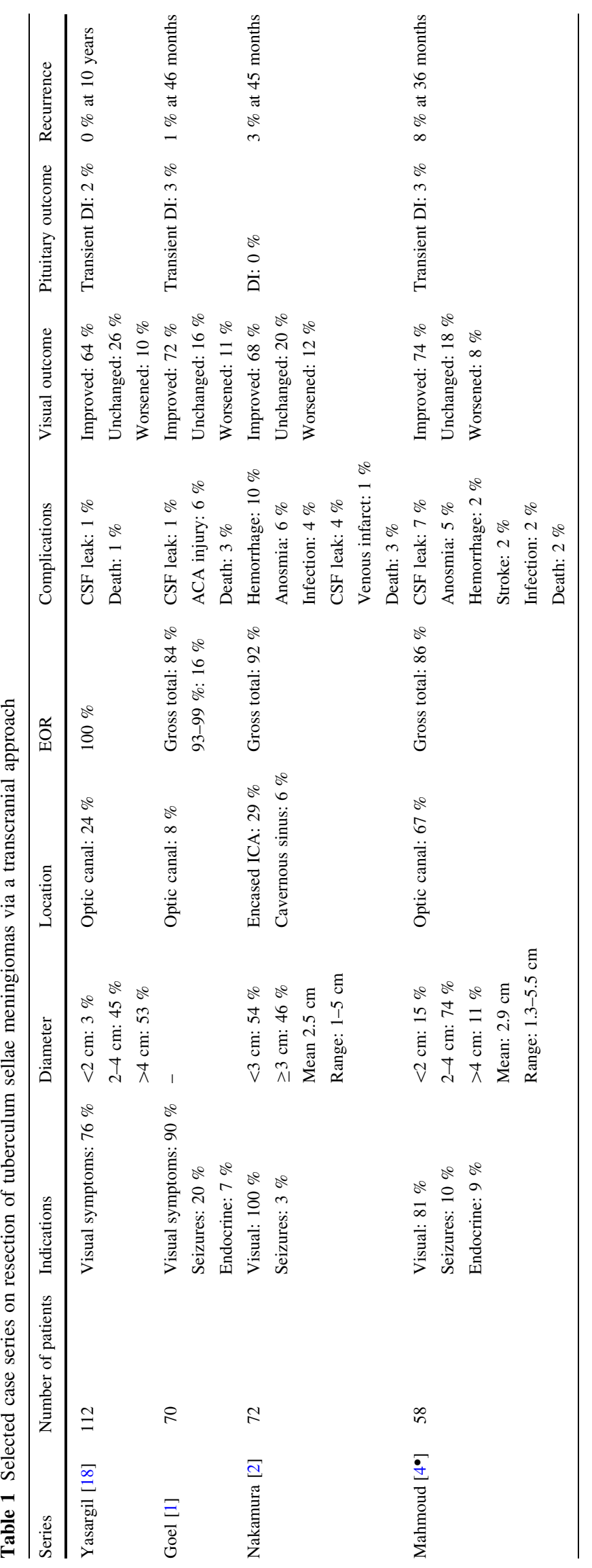



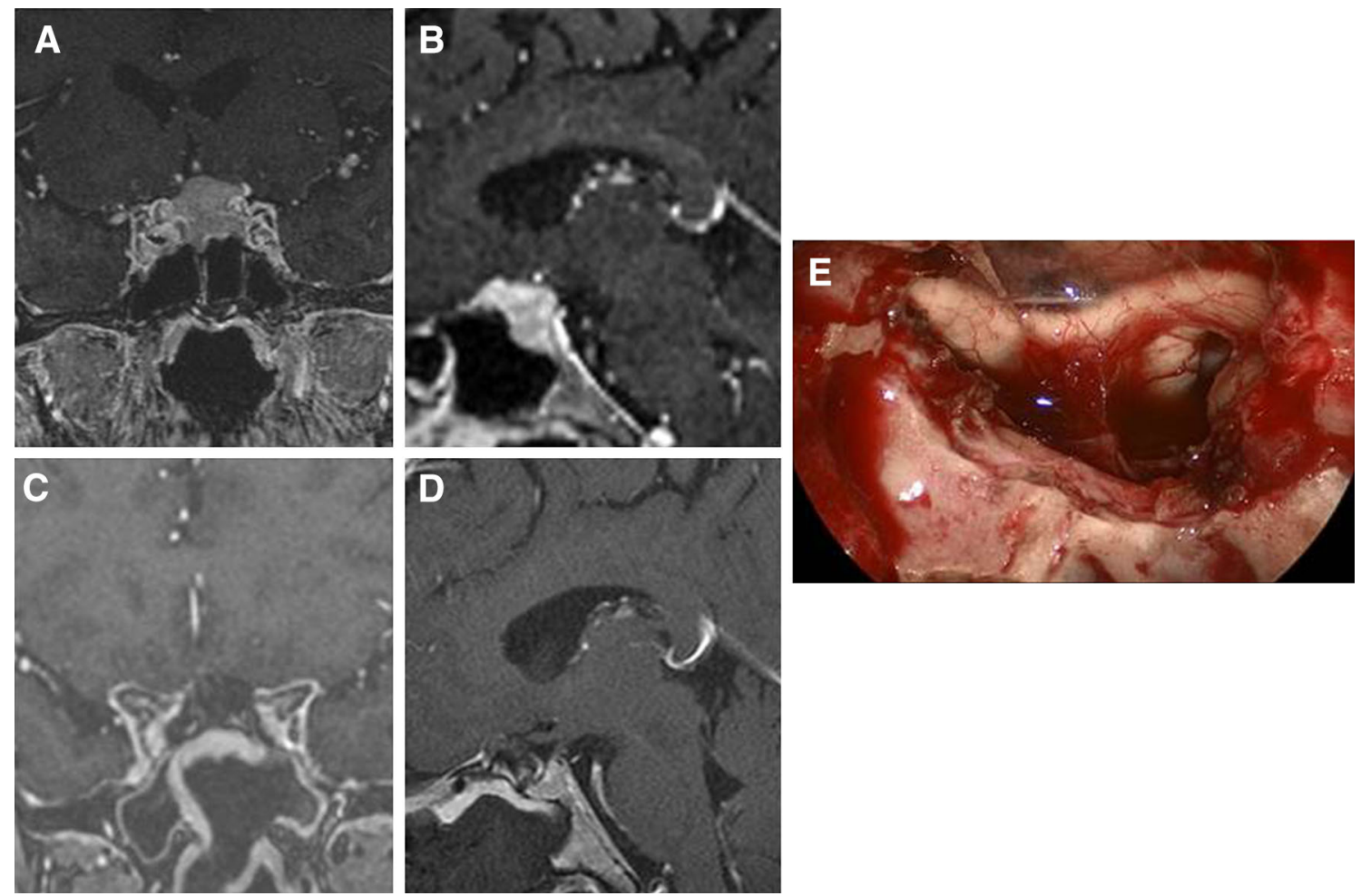

Fig. 2 Case example of tuberculum sella meningioma resected via endoscopic endonasal transtubercular approach. Panels $\mathbf{a}$ and $\mathbf{b}$ are preoperative contrast-enhanced MRI T1-weighted scans demonstrating a tuberculum sella meningioma with bilateral optic canal

then bipolared and opened in a cruciate fashion; the superior intercavernous sinus is often compressed and does not bleed. Once tumor is exposed, the dissection proceeds in such a fashion where the anterior lip is identified and dissected in an extracapsular fashion; this is typically the safest part given that this is least likely to involve any critical neurovascular structures. Then, dissecting along the lateral aspects of the tumor, the optic nerves are identified early in the canals and tumor is dissected away bilaterally. As tumor is debulked and dissected, an arachnoidal plane can be found along the lateral aspect of the tumor and followed posteriorly and superiorly; attention is paid to the superior-posterior aspect of the tumor where the A2 branches and recurrent artery of Heubner are separated away using sharp dissection techniques. Once the superior level of the dissection proceeds inferiorly toward the chiasm, the chiasm is then decompressed. It is essential during this stage to preserve the superior hypophyseal artery bilaterally and its branches to the chiasm and pituitary stalk. The anterior-inferior margin is then devascularized and detached by identifying the diaphragm sella-this can be resected if involved and can aid in the identification of the inferior aspect of the pituitary stalk. At this point, the inferior edge of the tumor is lifted, the entire length of the pituitary stalk dissected free and the membrane of Liliquist extension. Panels $\mathbf{c}$ and $\mathbf{d}$ are postoperative scans demonstrating a gross total resection. Panel $\mathbf{e}$ is an intraoperative image showing both optic nerves, the pituitary stalk and arachnoid covering the anterior communicating artery complex

is identified and preserved. This typically completes the resection. After copious irrigation, a multi-layer reconstruction consisting of multiple free grafts (i.e., fascia lata), rigid reconstruction (i.e., MedPor implant), and a vascularized nasoseptal flap is performed (Fig. 2).

Selected Case Series: Extent of Resection, Complications, Outcomes, and Recurrence

The largest cases series on the expanded endonasal approaches (EEA) for resection of TSMs are summarized in Table 2 [23-27]. These case series selected small TSMs (majority smaller than 3-4 cm) with limited medial optic canal involvement, with or without arterial encasement. In comparison to the published open series, the endoscopic series indicate a selection bias with regards to cases. Reported contraindications include extension lateral to the optic canals, superolateral tumor extension beyond the optic nerves, vascular encasement, and preoperative evidence of pial invasion (i.e., T2/flair signal change in adjacent brain, lack of "cortical cuff"). Gross total resection rates ranged from 67 to $92 \%$ and recurrence rates were approximately $5 \%$ over shorter mean follow-up time periods in comparison to the TCR series. Complications included CSF leak (range 0-25\%), stroke (0-1\%), and 


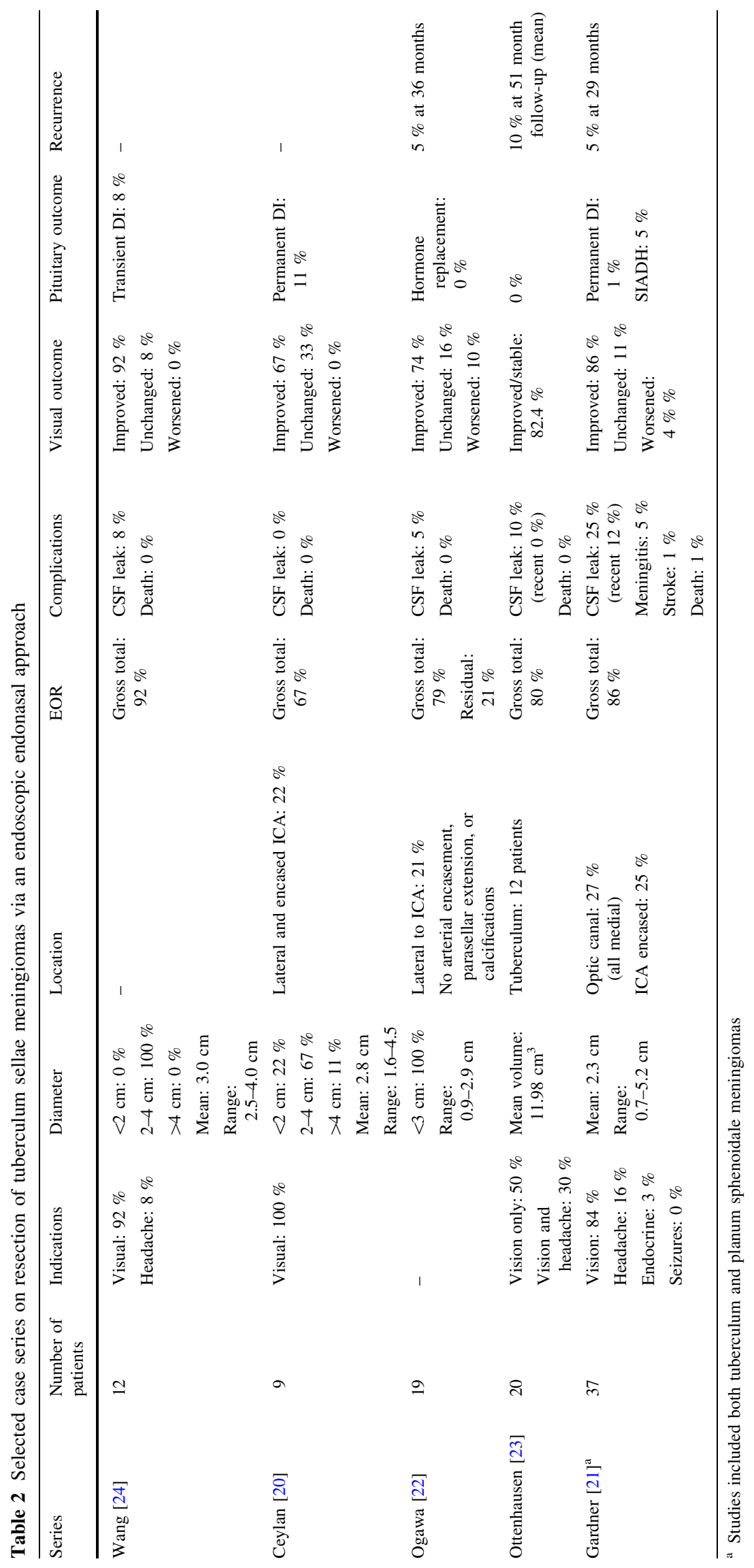


death $(0-1 \%)$. Postoperatively, vision improved in $67-92 \%$ of patients, remained stable in 8-33\% of patients, and worsened in $0-10 \%$ of patients. Permanent DI occurred in $0-11 \%$ of patients, and anterior pituitary dysfunction did not occur.

The Pittsburgh group [24] demonstrated that factors which significantly limited surgical outcomes where tumor size, vascular encasement, and the shape of the free surface of the tumor (smooth vs. multi-lobulated). Tumor size (mean diameter) at above 2.3 or $3 \mathrm{~cm}$ was significant-as opposed to the $4 \mathrm{~cm}$ delineation noted in the TCR series. A multi-lobular configuration was through to be reflective of more aggressive tumors with pial invasion and lack of an arachnoidal plane. The presence of vascular encasement decreased the GTR rate from 91.1 to $31.6 \%$. Overall, the published series indicate that in well-trained hands, endoscopic endonasal approaches provide appropriate outcomes in well-selected patients.

\section{Transcranial or Endoscopic Resection: Relative Indications}

With advances in microsurgical and endoscopic techniques, the optimum approach for resection of TSMs is greatly debated in the neurosurgical community. Historically, the TCA was the traditional route employed for the resection of these tumors. However, over the past decade, extended EEAs have become increasing utilized for ventral midline pathologies from the anterior skull base down to the clivus and upper cervical spine, including TSMs. As with most neurosurgical pathology, no controlled clinical trials have been done in order to determine the relative merits of each approach.

Two recently published meta-analyses compared the TCA and EEA for resection of TSMs and provide a framework for this discussion [28, 29]. In both pooled analyses, the rates of gross total resection (60-92\% in EEA cohort vs. 74-92 \% in TCR cohort), overall complications (11-43\% with EEA vs. 5-28 \% with TCR), and recurrence were not significantly different between the two approaches. Although, the follow-up periods were shorter with the EEA studies and could represent a bias. However, the rates of CSF leak (0-62\% vs. 0-8 \% with TCR) and postoperative pituitary dysfunction were significantly higher for the EEA. There was a trend toward higher rates of postoperative visual deterioration with TCR (0-24\% vs. 0-17\% with EEA) and higher rates of visual improvement (50-100\% vs. $25-78 \%$ ) with EEA. While these studies were well designed, they failed to fully evaluate the selection biases in the individual case series, such as a trend toward smaller, less complex tumors with EEA, differences in follow-up time. In addition, the inherent limitations of meta-analyses make it difficult to make an evidence-based comparison.

From the technical standpoint, each skull base approach has its own merits. Early devascularization is thought to be an advantage of endoscopic approaches for TSMs-given their arterial supply is commonly from the posterior ethmoidal arteries. However, with larger tumors, feeders are parasitized from adjacent A1 and A2 segments of the anterior cerebral arteries. With the EEA approaches, these feeders are encountered later than with TCR techniques. Similarly important is the management of critical neural structures. While the absence of brain retraction has been cited as a relative advantage of endonasal approaches, meticulous microsurgical techniques (i.e., arachnoidal opening, Sylvian fissure dissection, CSF relaxation, and dynamic brain retraction) and modern skull base approaches (i.e., orbital ridge osteotomy) have nearly eliminated the rates of retraction-related complications in the TCR approaches. With regards to the optic apparatus, it has been suggested by several studies that visual outcomes are better with EEA due to the limited nerve manipulation and the relative ease with which the superior hypophyseal arteries (and other arterial perforators to the optic nerves) can be identified and preserved. The TCR approaches are theoretically limited by difficulties in decompressing the contralateral nerve, access to tumor infero-medial to the ipsilateral optic nerve and the risk of optic nerve manipulation. On the other hand, EEA approaches are limited with regards to tumor resection superior and lateral to the optic nerves or laterally along the planum. Reconstruction has been a major critique of the EEA approaches with the significantly elevated CSF leak rates reported. An improved understanding of endonasal reconstruction techniques, the increasing use of vascularized flaps and rigid reconstruction have helped to dramatically reduce postoperative CSF leak rates in recent years. With a reduced fear of CSF leaks, the endonasal approaches can be pushed further with regards to bony resection; this was likely one of the limitations for the lower rates of GTR with earlier endoscopic series.

Given the data and the merits and limitations of the surgical options, we only consider endoscopic techniques for smaller tumors $(<2.5 \mathrm{~cm})$ with no evidence of vascular encasement or extension supero-lateral to the optic nerves. For more complex pathology requiring extensive microsurgical dissection, an orbitocranial craniotomy provides the necessary benefits for a safe and adequate resection.

A thorough comparison of the two approaches for resection of TSMs is still limited. Currently, only a handful of case series on the EEA exist in the literature. In addition, as the techniques, technology, and experience in the EEA grow, the indications and outcomes with this approach will change in the years to come. Further studies are required to directly compare the two approaches. 


\section{Conclusions}

Both the TCA and EEA for resection of TSMs have clear surgical advantages and disadvantages. Smaller tumors without lateral optic canal involvement and without significant lateral extension are favorable for resection via an EEA. Larger tumors or tumors with vascular encasement, lateral optic canal involvement or significant lateral extension are best managed with an open TCA.

\section{Compliance with Ethics Guidelines}

Conflict of Interest Shaan M. Raza, Sabih T. Effendi, and Franco DeMonte have nothing to disclose.

Human and Animal Rights and Informed Consent This article does not contain any studies with human or animal subjects performed by any of the authors.

\section{References}

Papers of particular interest, published recently, have been highlighted as:

- Of importance

1. Goel A, Muzumdar D, Desai KI. Tuberculum sellae meningioma: a report on management on the basis of a surgical experience with 70 patients. Neurosurgery. 2002;51:1358-63 discussion 63-4.

2. Nakamura M, Roser F, Struck M, Vorkapic P, Samii M. Tuberculum sellae meningiomas: clinical outcome considering different surgical approaches. Neurosurgery. 2006;59:1019-28 discussion 28-9.

3. DeMonte F. Surgical treatment of anterior basal meningiomas. J Neurooncol. 1996;29:239-48.

4. - Mahmoud M, Nader R, Al-Mefty O. Optic canal involvement in tuberculum sellae meningiomas: influence on approach, recurrence, and visual recovery. Neurosurgery. 2010;67:ons108-18 discussion ons18-9. Describes technique of transcranial resection of tuberculum sella meningiomas.

5. Louis DN, Ohgaki H, Wiestler OD, et al. The 2007 WHO classification of tumours of the central nervous system. Acta Neuropathol. 2007;114:97-109.

6. Kolles H, Niedermayer I, Schmitt C, et al. Triple approach for diagnosis and grading of meningiomas: histology, morphometry of Ki-67/Feulgen stainings, and cytogenetics. Acta Neurochir (Wien). 1995;137:174-81.

7. Oya S, Kim SH, Sade B, Lee JH. The natural history of intracranial meningiomas. J Neurosurg. 2011;114:1250-6.

8. Hashiba T, Hashimoto N, Izumoto $\mathrm{S}$, et al. Serial volumetric assessment of the natural history and growth pattern of incidentally discovered meningiomas. J Neurosurg. 2009;110:675-84.

9. - Hashimoto N, Rabo CS, Okita Y, et al. Slower growth of skull base meningiomas compared with non-skull base meningiomas based on volumetric and biological studies. J Neurosurg. 2012;116:574-80. Provides background information on natural history of skull base meningiomas.

10. Rhoton AL Jr. The sellar region. Neurosurgery. 2002;51: S335-74.
11. - Kulwin C, Schwartz TH, Cohen-Gadol AA. Endoscopic extended transsphenoidal resection of tuberculum sellae meningiomas: nuances of neurosurgical technique. Neurosurg Focus. 2013;35:E6. Describes technique of expanded endoscopic endonasal resection of tuberculum sella meningiomas.

12. Pamir MN, Ozduman K, Belirgen M, Kilic T, Ozek MM. Outcome determinants of pterional surgery for tuberculum sellae meningiomas. Acta Neurochir. 2005;147:1121-30 discussion 30.

13. Margalit NS, Lesser JB, Moche J, Sen C. Meningiomas involving the optic nerve: technical aspects and outcomes for a series of 50 patients. Neurosurgery. 2003;53:523-32 discussion 32-3.

14. Lee JH, Jeun SS, Evans J, Kosmorsky G. Surgical management of clinoidal meningiomas. Neurosurgery. 2001;48:1012-9 discussion 9-21.

15. Nozaki K, Kikuta K, Takagi Y, Mineharu Y, Takahashi JA, Hashimoto N. Effect of early optic canal unroofing on the outcome of visual functions in surgery for meningiomas of the tuberculum sellae and planum sphenoidale. Neurosurgery. 2008;62:839-44 discussion 44-6.

16. Sade B, Lee JH. High incidence of optic canal involvement in tuberculum sellae meningiomas: rationale for aggressive skull base approach. Surgical neurology. 2009;72:118-23 discussion 23.

17. Schick U, Hassler W. Surgical management of tuberculum sellae meningiomas: involvement of the optic canal and visual outcome. J Neurol Neurosurg Psychiatry. 2005;76:977-83.

18. Conway JE, Raza SM, Li K, McDermott MW, Quinones-Hinojosa A. A surgical modification for performing orbitozygomatic osteotomies: technical note. Neurosurg Rev. 2010;33:491-500.

19. Raza SM, Boahene KD, Quinones-Hinojosa A. The transpalpebral incision: its use in keyhole approaches to cranial base brain tumors. Expert Rev Neurother. 2010;10:1629-32.

20. Raza SM, Garzon-Muvdi T, Boaehene K, et al. The supraorbital craniotomy for access to the skull base and intraaxial lesions: a technique in evolution. Minim Invasive Neurosurg. 2010;53:1-8.

21. Yasargil MG. Microneurosurgery, vol IVB. Stuttgart: Thieme publishing; 1996.

22. Jane JA, Mc KW. Importance of failing vision in early diagnosis of suprasellar meningiomas. Br Med J. 1962;2:5-7.

23. Ceylan S, Koc K, Anik I. Extended endoscopic transphenoidal approach for tuberculum sellae meningiomas. Acta Neurochir (Wien). 2011;153:1-9.

24. Koutourousiou M, Fernandez-Miranda JC, Stefko ST, Wang EW, Snyderman CH, Gardner PA. Endoscopic endonasal surgery for suprasellar meningiomas: experience with 75 patients. J Neurosurg. 2014;120:1326-39.

25. Ogawa Y, Tominaga T. Extended transsphenoidal approach for tuberculum sellae meningioma-what are the optimum and critical indications? Acta Neurochir (Wien). 2012;154:621-6.

26. Ottenhausen M, Banu MA, Placantonakis DG, et al. Endoscopic endonasal resection of suprasellar meningiomas: the importance of case selection and experience in determining extent of resection, visual improvement, and complications. World Neurosurg. 2014;pii:S1878-8750.

27. Wang Q, Lu XJ, Ji WY, et al. Visual outcome after extended endoscopic endonasal transsphenoidal surgery for tuberculum sellae meningiomas. World Neurosurg. 2010;73:694-700.

28. Bohman LE, Stein SC, Newman JG, et al. Endoscopic versus open resection of tuberculum sellae meningiomas: a decision analysis. ORL J Otorhinolaryngol Relat Spec. 2012;74:255-63.

29. Clark AJ, Jahangiri A, Garcia RM, et al. Endoscopic surgery for tuberculum sellae meningiomas: a systematic review and metaanalysis. Neurosurg Rev. 2013;36:349-59. 